\title{
On the Regularity of Solutions to the Yamabe Equation and the Existence of Smooth Hyperboloidal Initial Data for Einstein's Field Equations
}

\author{
Lars Andersson $^{1, \star}$, Piotr T. Chruściel ${ }^{2, \star \star}$, and Helmut Friedrich ${ }^{3}$ \\ 1 Department of Mathematics, Royal Institute of Technology, S-100 44 Stockholm, Sweden \\ 2 Center for Mathematics and its Applications, Australian National University, Canberra ACT2601, \\ Australia \\ 3 Max-Planck Institut für Astrophysik, Karl-Schwarzschild-Strasse 1, \\ W-8046 Garching bei München, FRG
}

Received May 3, 1991; in revised form April 24, 1992

\begin{abstract}
The regularity of the solutions to the Yamabe Problem is considered in the case of conformally compact manifolds and negative scalar curvature. The existence of smooth hyperboloidal initial data for Einstein's field equations is demonstrated.
\end{abstract}

\section{Introduction}

In this paper we shall show the existence of smooth hyperboloidal initial data for Einstein's field equations. Such data occur under the following circumstances. Suppose one is given a solution to Einstein's source free field equations (of signature $(-,+,+,+))$ with cosmological constant $\Lambda \leq 0$ which is "asymptotically flat" respectively "asymptotically simple" in the sense of Penrose [15]. Let $\widetilde{M}$ be a spacelike hypersurface in this space-time which extends up to the conformal boundary $\mathscr{T}$. The latter is a null hypersurface if $\Lambda=0$ and time-like if $\Lambda<0$. If one assumes that $\widetilde{M}$ can be extended across $\mathscr{T}$ as a smooth space-like hypersurface in some smooth conformal extension of the given space-time and denotes by $\partial M$ the intersection of this extension with $\mathscr{Y}$, then $M=\widetilde{M} \cap \partial M$ will be a smooth compact manifold with boundary.

We call the triple $(\widetilde{M}, \tilde{g}, \tilde{\chi})$, where $\tilde{g}$ is the Riemannian metric and $\tilde{\chi}$ the second fundamental form induced on $\widetilde{M}$ by the space-time metric, a "hyperboloidal initial data set." Since this initial data set has been obtained from a solution to the Einstein equations the fields $\tilde{g}$ and $\tilde{\chi}$ satisfy the constraint equations. Because of the specific geometric situation which is represented by this data set and because it has been derived from a space-time admitting a smooth conformal boundary $\mathscr{T}$ at infinity the fields $\tilde{g}$ and $\tilde{\chi}$ satisfy certain special smoothness respectively fall-off conditions at $\partial M$.

\footnotetext{
* Supported in part by NFR, the Swedish Academy of Sciences and the Gustavsson Foundation $\star \star$ On leave from the Institute of Mathematics of the Polish Academy of Sciences, Warsaw
} 
The first and most fundamental of these is an immediate consequence of the definition of the conformal boundary $\mathscr{T}$. There exist on $M$ a smooth Riemannian metric $h$ and a smooth defining function $\Omega$ of $\partial M$ such that

$$
\tilde{g}=\Omega^{-2} h \text { on } \widetilde{M} .
$$

The second condition is more complicated to analyse and to explain. For this reason we study in this paper the class of data satisfying the condition

$$
\tilde{\chi}=\frac{1}{3} \operatorname{tr}_{\tilde{g}}(\tilde{\chi}) \tilde{g}
$$

This is similar to the condition of time-symmetry for standard Cauchy data which are asymptotically flat at space-like infinity. The existence of hyperboloidal initial data which do not necessarily satisfy (1.2) will be discussed by two of us (L. A. and P. T. C.) in a subsequent paper [2].

The momentum constraint equations and our geometric assumptions now imply that $\operatorname{tr}_{\tilde{g}}(\tilde{\chi})=$ const $(\neq 0$ in the case $\Lambda=0$ ) and the Hamiltonian constraint equation takes, possibly after rescaling of $\tilde{g}$ by a constant conformal factor, the form $R(\tilde{g})=-6$. We consider now the tensor fields

$$
\begin{aligned}
\Omega, t & =\frac{1}{3} D_{\imath} D^{i} \Omega, \\
s_{i j} & =-\Omega^{-1}\left(D_{i} D_{j} \Omega-\frac{1}{3} h_{i j} D_{k} D^{k} \Omega\right), \\
d_{\imath j} & =\Omega^{-1}\left(R_{i j}(h)-\frac{1}{3} h_{i j} R(h)-s_{i j}\right),
\end{aligned}
$$

which are derived on $\widetilde{M}$ by using $\Omega, h$ as well as the covariant derivative operator $D$ and the Ricci tensor defined by $h$. A discussion of the origin and meaning of these expressions and their generalizations to the case where (1.2) is not imposed may be found in the paper [6] and in the references given therein. Let us note here that the tensor field $C_{i j}=\Omega d_{i j}$ is the electric part of the conformal Weyl tensor on $\widetilde{M}$ of the corresponding 4-dimensional metric and that it contains all the information about the conformal Weyl tensor on $\widetilde{M}$ because the magnetic part of the Weyl tensor vanishes under our hypothesis (1.2). It has been shown by Penrose in [15] that the Weyl tensor vanishes on $\mathscr{T}$ where the latter is smooth and admits spherical sections. Therefore we say that the solution $(\widetilde{M}, \tilde{g}, \tilde{\chi})$ of the constraint equations for which the relations (1.1), (1.2) are satisfied, is a "smooth hyperboloidal initial data set" if the tensor fields (1.3), (1.4), (1.5) extend to smooth tensor fields on all of $M$. It may be emphasized here that we do not impose the condition that $\partial M$ be homeomorphic to a union of 2-spheres.

It is our aim to construct general smooth hyperboloidal initial data satisfying (1.2) from suitable chosen "free data." The discussion above suggests constructing the hyperboloidal initial data as follows. Give as free data an arbitrary 3-dimensional, oriented, compact, smooth Riemannian space $(M, h)$ with boundary $\partial M$. On $M$ choose a defining function $\varrho$ of $\partial M$, i.e. a smooth function $\varrho$ which is positive on $\widetilde{M}$ and satisfies on $\partial M$ the conditions $\varrho=0, d \varrho \neq 0$. Find a smooth function $u$ on $\widetilde{M}$ such that

$$
R\left(u^{4} \varrho^{-2} h\right)=-6 \quad \text { and } \quad u \geq u_{0}=\text { const }>0 \text { on } \widetilde{M} .
$$


Then $\left(\widetilde{M}, \tilde{g}=u^{4} \varrho^{-2} h, \tilde{\chi}=\frac{1}{3} \operatorname{tr}_{\tilde{g}}(\tilde{\chi}) \tilde{g}\right)$, with suitable defined constant $\operatorname{tr}_{\tilde{g}}(\tilde{\chi})$, is a solution of the constraint equations which satisfies (1.1) with $\Omega=u^{-2} \varrho$. It qualifies as a smooth hyperboloidal initial data set only if we can show the smooth extensibility of the tensor fields (1.3), (1.4), (1.5).

As will be discussed in detail below the first equation of (1.6), if expressed with respect to the metric $h$, is an elliptic partial differential equation which degenerates on $\partial M$. Thus we have to show the existence of a solution of this equation which is smooth and positive up to the boundary on $M$ and on top of this we have to demonstrate the smooth extensibility of the tensor fields (1.3), (1.4), (1.5). This is a highly overdetermined problem which suggests that our data are not so free as assumed. It is not clear a priori which conditions have to be imposed on the data $(M, h)$ such that they lead to smooth hyperboloidal data. The requirements turn out to be surprisingly simple and geometrical.

Theorem 1.1. Suppose $(M, h)$ is a 3-dimensional, orientable, compact, smooth Riemannian space with boundary $\partial M$. Then there exists a unique solution $u$ of (1.6) and the following conditions are equivalent.

1. The function $u$ as well as the tensor fields (1.3), (1.4), (1.5), determined on $\widetilde{M}$ from $h$ and $\Omega=u^{-2} \varrho$ extend smoothly to all of $M$.

2. The conformal Weyl tensor $C_{i j}=\Omega d_{i j}$ goes to zero at $\partial M$.

3. The conformal class of $h$ is such that the condition

$$
\lambda^{*}=0 \text { on } \partial M
$$

is satisfied, where $\lambda^{*}$ denotes the trace-free part of the second fundamental form implied by the metric $h$ on $\partial M$.

Theorem 1.1 will be proved in Sect. 5 as a consequence of Theorems 1.2 and 1.3 discussed below.

The discussion of condition 2 of Theorem 1.1 in Sect. 5 shows how the smoothness of the conformal initial data set at $\partial M$ is related under our assumptions to the falloff behaviour of the conformal Weyl tensor at $\partial M$. Condition 3 tells us how we have to choose the "free data" $(M, h)$ to construct smooth hyperboloidal data. Apart from our simplifying assumption on the second fundamental form $\tilde{\chi}$ on $\widetilde{M}$ (which is relaxed in [2]) these data are as general as we can expect. It is a remarkable fact that the analysis of the constraint equation on the hypersurface $M$ together with the smoothness requirements do not yield more stringent conditions than already known from the analysis of the full Einstein equations in a neighbourhood of the conformal boundary $\mathscr{T}:$ In the case $\Lambda=0$ condition (1.7) is equivalent to the well known property that $\mathscr{T}$ is shear-free on $\partial M$.

In the case of a cosmological constant $\Lambda<0$ our result opens the possibility to investigate the existence of asymptotically simple solutions to Einstein's field equations by analysing initial boundary value problems for the regular conformal Einstein equations (cf. [7]) where initial data are prescribed on a hyperboloidal hypersurface and boundary data are given on the time-like conformal boundary $\mathscr{T}$. For an application in the case $\Lambda=0$ the reader is referred also to the article [7].

From the point of view of these applications it is of interest that the result on the smoothness of the tensor fields (1.3), (1.4), (1.5) is obtained by imposing conditions on the "free data" only on $\partial M$. Moreover, the proof of the smoothness requires the discussion of $u$ only in an arbitrary small neighbourhood of $\partial M$. This suggests that 
the analysis will generalize in particular to the case where massive sources are present which have spatially compact support in the physical space-time. It is also remarkable that besides the orientability of $M$, which we assumed, no further conditions on the topology of $M$ enter the discussion.

To avoid misunderstanding it may be remarked that the result of Theorem 1.1 leaves, of course, open the question under which circumstances in the case $\Lambda=0$ smooth hyperboloidal data can arise by Einstein evolution from asymptotically euclidean standard Cauchy data for Einstein's equations. We hope, however, that our analysis and its subsequent generalization will contribute to the understanding of this question.

We turn now to a discussion of the technical results underlying the proof of Theorem 1.1. The problem of constructing solutions to the constraint equations implied by Einstein's fields equations on space-like hypersurfaces is closely related to the Yamabe problem. In fact, finding a solution $u$ to (1.6) is a Yamabe problem and the first of conditions (1.6), if written as a partial differential equation for the unknown $u$, is the special case of the Lichnerowicz equation which is known now as the Yamabe equation. In various contexts the investigation of this special case has been observed to be crucial for the understanding of the properties of the solutions to the Lichnerowicz equation in general.

The Yamabe problem in the category of compact Riemannian manifolds has received its solution in the paper [16] by Schoen. The Yamabe problem in the case of an open manifold has been studied under various assumptions on the structure of the manifold and on the sign of the Ricci scalar. The following result outlines the situation which we shall analyse in Sect. 3 and 4 of this article.

Theorem 1.2. Let $(M, h)$ be a smooth, orientable Riemannian manifold of dimension $n \geq 3$ with metric $h$ and compact manifold $M$ with boundary $\partial M$. Suppose that $\tilde{R}$ is a negative real number. Then there exists a unique smooth function $w$ such that $w$ is positive on the interior $\widetilde{M}$ of $M$, satisfies $w(p) \rightarrow \infty$ as $p \rightarrow \partial M$, and the metric $\tilde{g}$ obtained on $\widetilde{M}$ from $h$ by the conformal rescaling $\tilde{g}=w^{4 /(n-2)} h$ has Ricci scalar $\tilde{R}$.

Moreover, if $\varrho$ is a smooth defining function for the boundary of $M$, such that $\varrho$ is positive on $\widetilde{M}$ while $\varrho=0$ and $|d \varrho|_{h}=1$ on $\partial M$, the function $w$ satisfies $w \varrho^{(n-2) / 2} \rightarrow(-n(n-1) / \tilde{R})^{(n-2) / 4}$ as $p \rightarrow \partial M$ and thus the space $(\widetilde{M}, \tilde{g})$ is complete.

A similar result has been obtained by Loewner and Nirenberg [9] under simplifying assumptions on $(M, h)$ and the existence part has been shown in general by Aviles and McOwen [3,4]. These authors also studied situations which in various respects were more general than the one described above. The uniqueness part of the theorem will be discussed in this paper and the solution will be studied in detail.

It is clear that the rescaled metric would not have the completeness property if the function $w$ would remain bounded along a sufficiently smooth curve which ends on the boundary. We leave it as an open question whether the requirement of the completeness of $\tilde{g}$ and the condition on its Ricci scalar implies that in fact $w$ goes to infinity at the boundary; such a result has been announced without proof in [12]. The answer to this question is, however, not important for the application we shall study later on.

One of the main results of this paper is a careful analysis of the behaviour of the solution $w$ near $\partial M$. It is easy to see that by a rescaling of $\tilde{g}$ with a suitable constant conformal factor we can achieve $\tilde{R}=-n(n-1)$. This value of $\tilde{R}$ will be assumed in the following. For our analysis it will be convenient to consider instead of $w$ the 
function $u=w \varrho^{(n-2) / 2}$ and to introduce the metric $g=\varrho^{-2} h$ on $\widetilde{M}$. The function $u$ is then the unique solution, positive and continuous on $M$, to the boundary value problem

$$
\begin{aligned}
L_{g} u+n(n-1) u^{(n+2) /(n-2)} & =0, \\
\left.u\right|_{\partial M} & =1,
\end{aligned}
$$

where $L_{g}$ denotes the conformally invariant Laplacian

$$
L_{g}=4 \frac{n-1}{n-2} \Delta_{g}+R(g)
$$

with $\Delta_{g}=-\operatorname{tr} \nabla d$ the Laplace-Beltrami operator and $R(g)$ the Ricci scalar defined by $g$.

As mentioned before, the Laplacian $L_{g}$ considered as an operator on the compact space $(M, h)$ is degenerate at the boundary. Therefore the classical regularity theory for boundary value problems does not apply. In fact, $\Delta_{g}$ is an operator of the kind considered in e.g. [11,13 or 1]. In the papers [11] and [13] the regularity of parametrices of elliptic $\mathscr{T}_{0}$-operators was considered, using a technique of microlocalizing "near the boundary," which as a byproduct gives statements about invertibility of $\Delta_{g}$ in a setting of weighted Sobolev spaces. In [1] the Fredholm and isomorphism property of certain elliptic systems was considered in a similar setting. In this case, the main tool was the use of the McKean inequality, rather than microlocal techniques. In the present paper we show how to get statements about the regularity of solutions to problems like (1.8) starting form the isomorphism property of the operator rather than from statements about the regularity of the parametrix. The main technical step is to get tangential regularity by commuting $\mathscr{W}_{b}$ operators through the equation, see Sect. 4. This approach is quite elementary and it appears easier to apply in the case of systems, where the analysis of the parametrix can be rather cumbersome, see [10] for an example.

Recently, the techniques developed by Melrose and Mazzeo have been independently applied to the type of problem considered in the present paper by Mazzeo, see [12]. The theme of that paper is the polyhomogeneity of solutions to the general singular Yamabe problem, i.e. with lower dimensional boundary.

The most important technical results of the present paper are stated in the following theorem. Here the equality in (1.10) is to be understood in terms of polyhomogeneous expansions (cf. Definition 2.5 below, where the notion of tangential smoothness is also discussed). To avoid any ambiguities, let us stress that in our convention $M$ is a compact manifold with its boundary included. Thus, e.g., $C^{\infty}(M)=C^{\infty}(\bar{M})$ is a space of functions which are $C^{\infty}$ up to the boundary.

Theorem 1.3. On the manifold $(\widetilde{M}, g)$, let $u \in C^{0}(M) \cap C^{\infty}(\widetilde{M})$ be the unique positive solution to the boundary value problem (1.8) and (1.9), which exists by Theorem 1.2. Then:

1. $u$ has an asymptotic expansion

$$
u=1+\sum_{i=1}^{\infty} \sum_{j=0}^{N_{\imath}} u_{i j}(\log \varrho)^{j} \varrho^{i}
$$


at $\partial M$ with functions $u_{i j} \in C^{\infty}(\partial M)$ and nonnegative integers $N_{i}$ such that $N_{i}=0$ for $i<n=\operatorname{dim} M$. Furthermore $u$ is tangentially smooth.

If $N_{n}=0$, then in fact $N_{i}=0$ for all $i$ and $u \in C^{\infty}(M)$.

2. For a given metric $h$ there always exist smooth defining functions $\varrho$ as considered above such that $R(g)=-n(n-1)+\varrho^{n} R_{n}$ near $\partial M$ with $R_{n} \in C^{\infty}(M)$. The solution $u \in C^{\infty}(M)$ if and only if

$$
R_{n}=0 \text { on } \partial M
$$

This condition describes the property of the (smooth) conformal class of the metric $h$ which decides on the smoothness of the function $u$ at $\partial M$.

3. In the case of dimension $n=3$ we define on $\partial M$ the function

$$
C(h, \partial M, M)=\delta \cdot \delta \cdot \lambda^{*}+\lambda^{*} \cdot R^{*}-\frac{1}{2} \operatorname{tr}(\lambda) \lambda^{*} \cdot \lambda^{*},
$$

where $\lambda^{*}$ denotes the trace free of the second fundamental form $\lambda$ induced by $h$ on $\partial M, R^{*}$ the orthogonal projection of $\operatorname{Ric}(h)$ on $\partial M$, and the first term on the righthand side of (1.12) is the double divergence of $\lambda^{*}$ with respect to the inner covariant derivative on $\partial M$ (cf. Sect. 5). This function is a conformal density of weight -3 and

$$
u \in C^{\infty}(M) \text { if and only if } C=0 \text { on } \partial M
$$

The first two parts of Theorem 1.3 will be proved in Sect. 4 and the third part in Sect. 5 where we also give an example of a smooth Riemannian manifold $(M, h)$ with boundary for which the quantity $C(h, \partial M, M)$ does not vanish everywhere on $\partial M$.

\section{Preliminaries}

\subsection{Differential Geometric Considerations}

We shall mainly be concerned with Riemannian manifolds of the following type:

Definition 2.1. Let $(M, h)$ denote an oriented, compact $C^{\infty}$ Riemannian manifold of dimension $n \geq 3$ with nonempty boundary $\partial M$ and interior $\widetilde{M}$. Assume that $\varrho \in C^{\infty}(M)$ denotes a defining function for $\partial M$, i.e. $\varrho>0$ on $\widetilde{M}$ while $\varrho=0$ but $d \varrho \neq 0$ everywhere on $\partial M$. Then the manifold $(\widetilde{M}, g)$, where $g=\varrho^{-2} h$, is said to be conformally compact.

Since $M$ is compact with boundary the statement $f \in C^{\infty}(M)$ means that $f$ as well as all its derivatives on $\widetilde{M}$ extend continuously to all of $M$.

It is easy to see that the Riemannian space $(\widetilde{M}, g)$ is complete.

The following formulae which express the curvature of $g$ in terms of fields derived from $\varrho$ and $h$ will be useful for us.

$$
\operatorname{Riem}(g)=\varrho^{-2}\left(\operatorname{Riem}(h)-h \times\left(-\varrho^{-1} \operatorname{Hess}_{h} \varrho+\frac{1}{2} \varrho^{-2}|d \varrho|_{h}^{2} h\right)\right),
$$

where Riem denotes the $(4,0)$ form of the Riemann tensor and $\times$ denotes the Kulkarni-Nomizu product, see [5, 1.110]. It follows from this formula that $g$ has 
asymptotically negative sectional curvature: $K_{g}(p) \rightarrow-|d \varrho|_{h}(q)$ as $p \rightarrow q \in \partial M$. Taking contractions, we get

$$
\begin{gathered}
\operatorname{Ric}(g)=\operatorname{Ric}(h)+\varrho^{-1}\left[(n-2) \operatorname{Hess}_{h} \varrho-\left(\Delta_{h} \varrho\right) h\right]-(n-1) \varrho^{-2}|d \varrho|_{h}^{2} h, \\
R(g)=-n(n-1)|d \varrho|_{h}^{2}-(2 n-2) \varrho\left(\Delta_{h} \varrho\right)+\varrho^{2} R(h) \\
\widetilde{\operatorname{Ric}}(g)=\widetilde{\operatorname{Ric}}(h)+\frac{n-2}{\varrho}\left(\operatorname{Hess}_{h} \varrho+\frac{\Delta_{h} \varrho}{n} h\right)
\end{gathered}
$$

where for any metric $k$ the symbol $\widetilde{\operatorname{Ric}}(k)$ denotes the trace-free part of its Ricci tensor.

Let $h$ be a metric, $\Theta$ a positive function, and set $\tilde{h}=\Theta^{4 /(n-2)} h$. The conformally covariant Laplacian of $h, L_{h}=\kappa \Delta_{h}+R(h)$ with $\kappa=4(n-1) /(n-2)$, is related to the Ricci scalar of $\tilde{h}$ by

$$
L_{h} \Theta=\Theta^{(n+2) /(n-2)} R(\tilde{h})
$$

The conformal covariance of the operator $L_{h}$ is expressed by the identity

$$
L_{h} u=\Theta^{(n+2) /(n-2)} L_{\tilde{h}}\left(\Theta^{-1} u\right)
$$

which holds for all sufficiently smooth functions $u$.

Suppose that $(\widetilde{M}, g)$ is a conformally compact Riemannian manifold with $g=\varrho^{-2} h$ as described in Definition 2.1. Furthermore, assume that $u \in C^{0}(M) \cap C^{\infty}(\bar{M})$ is a positive solution to the boundary value problem (1.8), (1.9). Thus the Ricci scalar of the metric $\tilde{g}=u^{4 /(n-2)} g$ takes the value $R(\tilde{g})=-n(n-1)$. By (2.6) these conditions will remain preserved under transformations $h \rightarrow \Theta^{4 /(n-2)} h$ (respectively $\left.\varrho \rightarrow \Theta^{-2 /(n-2)} \varrho\right)$ and $u \rightarrow \Theta^{-1} u$, where $\Theta$ is a positive smooth function on $M$ with $\Theta=1$ on $\partial M$. In what follows we shall exploit this freedom at various occasions without mentioning it each time.

The analysis of the behaviour of the solution $u$ near the boundary is done most conveniently in terms of functions, frame fields, and a choice of conformal scaling which are well adapted to the boundary. Let $(M, h, \varrho, g)$ be given as in Definition 2.1. Denote by $x$ the smooth function, defined in an open neighbourhood $U$ of $\partial M$, which satisfies $x=0$ on $\partial M,|d x|_{h}^{2}=1$ on $U$, and for which $\operatorname{grad}_{h} x$ is inward pointing on $\partial M$.

Let $x_{0}$ be a positive number. We denote by $\Phi$ the map which sends a point $\left(p, x^{\prime}\right) \in \partial M \times\left[0, x_{0}\right.$ [ onto the point $q$ on the integral curve of $\operatorname{grad}_{h} x$ through $p$ at which the function $x$ takes the value $x^{\prime}$. For $x_{0}$ chosen sufficiently small this map is well defined and in fact a diffeomorphism of $\partial M \times\left[0, x_{0}\right.$ [ onto an open neighbourhood of $\partial M$. We assume $U$ to coincide with such a neighbourhood. We write $\Phi^{-1}(q)=\left(x^{\prime}, p\right)$, consider $(x, p)$ with $0 \leq x<x_{0}, p \in \partial M$ as "Gauss coordinates" on $U$, and call $U$ a "Gauss domain." For $s \in\left[0, x_{0}\right.$ [ we set $M_{s}=\{p \in U \mid x(p)=s\}$.

Let $\left\{e_{a}\right\}_{a=1, \ldots, n}$ denote a smooth orthonormal frame with respect to $h$, defined near a given point of $\partial M$, parallely propagated along the integral curves of $\operatorname{grad}_{h} x$, and such that $e_{1}=\operatorname{grad}_{h} x$. Such a frame will be called a "Gauss frame" in the following. We denote by $\Gamma_{a c}^{b}$ the connection coefficients with respect to this frame and assume in the following, that tensor indices refer to this frame. 
The vector fields $e_{A}, A=2, \ldots, n$ define a frame on each hypersurface $M_{s}$. With respect to this frame the coefficients of the second fundamental form $\lambda$ induced by $h$ on the hypersurface $M_{s}$ are given by $\lambda_{A B}=\Gamma_{A B}^{1}$. The components in the frame $\left\{e_{a}\right\}$ of the covariant derivative of a tensor field $T$ in the direction of $e_{1}$ are obtained by taking the ordinary derivatives of the components of $T$ with respect to the parameter $x$ along the integral curves of $\operatorname{grad}_{h} x$. Thus tensors can be conveniently analysed in terms of power series in $x$ near $\partial M$. We denote covariant differentiation in the direction of $e_{1}$ by $D_{x}$.

The Laplace-Beltrami operator defined by $h$ takes on $U$ the form

$$
\Delta_{h} u=-D_{x}^{2} u+\Delta_{k} u+\operatorname{tr}(\lambda) D_{x} u,
$$

where $\Delta_{k}$ denotes the Laplace-Beltrami operator defined by the interior metric $k$ induced by $h$ on the slice $M_{s}$ if $\Delta_{h}$ is considered at a point with $x=s$.

Lemma 2.1. Let $(M, h)$ be given as in Definition 2.1. Then

1. A defining function $\varrho$ for $\partial M$ can be chosen such that the Ricci scalar of the metric $g=\varrho^{-2} h$ satisfies

$$
R(g)=-n(n-1)+R_{n} \varrho^{n}
$$

with $R_{n} \in C^{\infty}(M)$.

2. In addition to this we can assume, possibly after rescalings of $\varrho$ and $h$ by smooth positive factors $\left(\varrho \rightarrow \Theta \varrho, h \rightarrow \Theta^{2} h\right)$, that

$$
|d \varrho|_{h}=1 \text { whence } \varrho=x \text { near } \partial M
$$

3. With this choice of $\varrho$ and scaling of $h$

$$
R(g)=x^{2} R(h)-2(n-1) x \operatorname{tr} \lambda-n(n-1) \quad \text { near } \partial M
$$

from which follows in particular that

$$
\left.\operatorname{tr}(\lambda)\right|_{\partial M}=0
$$

Proof. By choosing $\varrho$ with $|d \varrho|_{h}=1$ on $\partial M$ it follows from (2.3) that $R(g)=$ $-n(n-1)$ on $\partial M$. Taking formal derivatives of the same equation and restricting to $\partial M$ gives for $k \geq 1$ relations

$$
\left.D_{x}^{k} R(g)\right|_{\partial M}=2(n-1)\left(\varrho D_{x}^{k+2} \varrho-(n-k) D_{x} \varrho D_{x}^{k+1} \varrho\right)+F_{k},
$$

where $F_{k}$ depends on the functions $D_{x}^{j} \varrho$ with $j \leq k$ and interior derivatives of these on $\partial M$. Thus we can determine a defining function $\varrho$ with derivatives $D_{x}^{k} \varrho$ for $1 \leq k \leq n$ on $\partial M$ such that $R(g)$ vanishes there up to order $n-1$. For given metric $h$ these derivatives are fixed uniquely and then also $\left.D_{x}^{n} R(g)\right|_{\partial M}$ is determined.

Let $\Theta$ be a smooth positive function on $M$. The metric $g$ remains unchanged if $h$ and $\varrho$ are replaced by $\hat{h}=\Theta^{2} h$ and $\hat{\varrho}=\Theta \varrho$ respectively. By our choice of $\varrho$ we can write $1-|d \varrho|_{h}=\varrho f$ with a smooth function $f$. The condition $|d \varrho|_{\hat{h}}=1$ takes thus the form

$$
2 \Theta\left\langle\operatorname{grad}_{h} \varrho, d \Theta\right\rangle+\varrho|d \Theta|_{h}^{2}=\Theta^{2} f .
$$


By the general theory of first order scalar PDE's there exists near $\partial M$ a unique smooth solution $\Theta$ to this equation such that $\Theta$ coincides on $\partial M$ with a given smooth positive function $\Theta_{0}$. This gives the first part of (2.9). From now on we omit hats on $\hat{h}$, etc., thus $|d \varrho|_{h}=1$. Define a vector field $X$ by $d \varrho=h_{i j} X^{i} d x^{\jmath}$; from $\left(h_{i j} X^{i} X^{j}\right)_{, k}=0$ it follows

$$
X^{i} X_{; i}^{k}=0
$$

which shows that the integral curves of $X$ are affinely parametrized geodesics (of the metric $h$ ) orthogonal to $\partial M$, and $h_{i j} X^{i} X^{j}=1$ implies that for $x$ small enough $\varrho$ is the affine parameter along those geodesics, hence $\varrho=x$.

Equation (2.10) follows with this scaling of $h$ and with $\varrho=x$ from Eq. (2.3) and Eq. (2.7). Equation (2.11) follows by applying $D_{x}$ to Eq. (2.10) and evaluating on $\partial M$.

Remark 2.1. The proof also shows that under the above rescaling of $\varrho$ and $h$ with $\Theta$ the function $\left.R_{n}\right|_{\partial M}$ scales with the factor $\Theta_{0}^{-n} \equiv\left(\left.\Theta\right|_{\partial M}\right)^{-n}$, whence the property that $\left.R_{n}\right|_{\partial M} \not \equiv 0$ is a property of the (smooth) conformal structure of $h$.

For later use we note the expression of the Laplace-Beltrami operator of $g$ in terms of $h$ and $\varrho$

$$
\Delta_{g} u=\varrho^{2} \Delta_{h} u+(n-2) \varrho\left\langle\operatorname{grad}_{h} \varrho, d u\right\rangle
$$

\subsection{Function Spaces and Operators}

Define the set $\mathscr{T}_{b}$ to be the set of smooth vector fields on $M$ which are tangent to $\partial M$ and define $\mathscr{T}$ to be the subset of smooth vector fields on $M$ which vanish on $\partial M$. Let $\left(y^{1}, y^{2}, \ldots, y^{n}\right)$ be a coordinate system defined in a neighbourhood of a point of $\partial M$ such that $y^{1}=0$ on $\partial M$ and denote $y^{1}$ also by $x$. Then the $\mathscr{T}_{b}$ and the $\mathscr{T}$ vector fields have local expressions

$$
Y=Y^{1}\left(x \partial_{y^{1}}\right)+Y^{2} \partial_{y^{2}}+\cdots+Y^{n} \partial_{y^{n}}
$$

respectively

$$
Y=Y^{1}\left(x \partial_{y^{1}}\right)+Y^{2}\left(x \partial_{y^{2}}\right)+\cdots+Y^{n}\left(x \partial_{y^{n}}\right)
$$

with $Y^{i} \in C^{\infty}$. Following Melrose and Mazzeo we introduce the space of operators (9) $\mathscr{P}_{b}$ (considered in [14]) and its subspace $\mathscr{O} \mathscr{P}_{0}$ (considered in [11] and [13]) generated by vector fields in $\mathscr{V}_{b}$ and $\mathscr{T}$, respectively. These spaces of operators can of course be extended to include corresponding pseudo-differential operators, but we will not need this in the present context.

For given integers $m \geq 1$ and $\beta \geq 0$ we denote by $\mathscr{O} \mathscr{P}_{b}^{m, \beta}$ the subset of operators of order $m$ in $\mathscr{O P}_{b}$, which in the coordinates considered above have local expression

$$
A=\sum_{|\alpha| \leq m} a_{\alpha} x^{\alpha_{1}+\beta} \partial_{y}^{\alpha}, \quad a_{\alpha} \in C^{\infty}
$$


where we have used standard multiindex notation such that e.g. $\partial_{y}^{\alpha}=\partial_{y^{1}}^{\alpha_{1}} \cdots \partial_{y^{n}}^{\alpha_{n}}$.

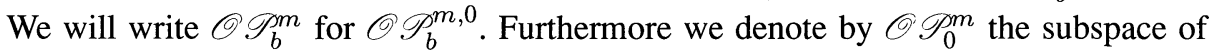
operators of order $m$ in $\mathscr{O} \mathscr{P}_{0}$, which have local expressions

$$
A=\sum_{|\alpha| \leq m} a_{\alpha} x^{|\alpha|}\left(\partial_{y}\right)^{\alpha}, \quad a_{\alpha} \in C^{\infty}
$$

Let us recall some definitions of function spaces over $\widetilde{M}$. Because of the completeness of the space $(\widetilde{M}, g)$ and the behaviour of its sectional curvature near $\partial M$, it is easily seen that there exists $a \in \mathbb{R}, a>0$ such that for any $x \in \widetilde{M}$ the set

$$
\bar{B}_{a}(x)=\left\{y \in M \mid d_{g}(x, y) \leq a\right\}
$$

is a convex normal neighbourhood of $x$. For tensor fields $u, g$ on $\widetilde{M}$ and given points $x \in \widetilde{M}, y \in \bar{B}_{a}(x)$ we denote by $\tilde{u}(x), \tilde{u}(y)$, and $\tilde{g}$ the corresponding tensors as $x$ respectively $y$ given by their components with respect to a normal frame associated with a normal neighbourhood centered at $x$. Set $a(x) \equiv a$, and define a space $C_{\delta}^{s, \alpha}(M, g)$ as follows:

Definition 2.2. For non-negative integers $s$ and real numbers $\alpha, \delta$ with $0<\alpha<1$, let the weighted Hölder space $C_{\delta}^{s, \alpha}(M, g)$ be the space of tensors on $\widetilde{M}$ which are locally of $C^{s, \alpha}$ differentiability class, for which the norm

$$
\|u\|_{C_{\delta}^{s, \alpha}(M, g)}=\max _{k \leq s}\left\|\nabla_{k} u\right\|_{C_{\delta}^{0, \alpha}(M, g)}
$$

is finite, where

$$
\|u\|_{C_{\delta}^{0, \alpha}(M, g)}=\sup _{x \in \widetilde{M}} \varrho^{-\delta}|u|_{g}+\sup _{x \in \widetilde{M}} \varrho^{-\delta}\left(\sup _{d_{g}(x, y) \leq a(x) ; x \neq y} \frac{|\tilde{u}(x)-\tilde{u}(y)|_{\tilde{g}}}{d_{g}(x, y)^{\alpha}}\right),
$$

and where $\nabla_{k} u$ denotes the covariant differential of order $k$ of $u$ with respect to the covariant derivative $\nabla$ defined by $g$ and $|.|_{g}$ denotes the pointwise tensor norm with respect to $g$.

Remark 2.2. 1. Note that these spaces are the same as the spaces used in [1] but with opposite sign on $\delta$.

2. We denote by $C_{\delta}^{s}(M, g)$ the spaces defined by norms similar to those given in (2.13), where, however, the second term on the right of (2.14) is dropped now. 3. In the following we set $C_{\delta}^{\infty}=\bigcap_{s=0}^{\infty} C_{\delta}^{s}$ and use analogous conventions for other
weighted spaces.

We can characterize the Hölder spaces above also by norms which are given in terms of the metric $h$. For a section $u$ of $T_{m}^{r}(M)$ the tensor norm with respect to $g$ is also given by $|u|_{g}=\varrho^{m-r}|u|_{h}$. To avoid the factors $\varrho^{m-r}$ we shall in the following only consider the case $m=r=0$. To emphasize the distinction of the, covariant 
derivatives involved we shall denote by $D$ the covariant derivative defined by $h$. The following weighted Hölder norms are equivalent to the norms (2.13)

$$
\|u\|_{C_{\delta}^{s, \alpha}(M, h)}=\max _{k \leq s}\left\|D_{k} u\right\|_{C_{\delta-k}^{0, \alpha}(M, h)}
$$

where the norms $\|u\|_{C_{\delta}^{0, \alpha}(M, h)}$ are defined as in (2.14) with $g$ replaced by $h$ and $a(x)=\min \left(\operatorname{rad}, d_{h}(x, \partial M)\right) / 2$, where $\operatorname{rad}$ is the injectivity radius of $h$. In an obvious way we get equivalent norms for the spaces $C_{\delta}^{s}(M, g)$. For a review of various properties of the weighted spaces, cf. e.g. [8].

Definition 2.3. We denote by $C_{\delta, b}^{s}$ the space

$$
C_{\delta, b}^{s}=\left\{u \in C_{\delta}^{s} \mid D_{Y_{1}} \cdots D_{Y_{k}} u \in C_{\delta}^{s-k}, 0 \leq k \leq s, Y_{i} \in \mathscr{V}_{b}\right\}
$$

Definition 2.4. Let $(M, h, \varrho)$ be as above. For given real numbers $a, \alpha$ with $a<\alpha$ we denote by $W_{\alpha}^{a}$ (respectively $W_{\alpha, b}^{a}$ ) the set of functions $f \in C^{\infty}(\widetilde{M})$ which have the following property: there exist non-negative integers $N, N_{\imath}, i=1, \ldots, N$ and real numbers $s_{0}=a<s_{1}<s_{2}<\cdots<s_{N}$ with $s_{N}<\alpha$ and there exists a smooth defining function $\varrho$ of $\partial M$ as well as functions $f_{i j} \in C^{\infty}(M)$ for $i=0,1, \ldots, N$ and $j=0,1, \ldots, N_{i}$ satisfying

$$
\left\langle d f_{i j}, \operatorname{grad}_{h} \varrho\right\rangle=0 \quad \text { near } \quad \partial M
$$

such that

$$
f=\sum_{i=0}^{N} \sum_{j=0}^{N_{\imath}} f_{i j}(\log \varrho)^{\jmath} \varrho^{s_{\imath}}+g
$$

with some function $g \in C_{\alpha}^{\infty}$ (respectively $g \in C_{\alpha, b}^{\infty}$ ). For given $c, a<c \leq s_{N}$, we denote by $W_{\alpha, b}^{a, c}$ the set of functions $f \in W_{\alpha, b}^{a}$ for which $N_{i}=0$ for $s_{i}<c$.

Condition (2.16) makes precise the sense in which $f_{i j} \in C^{\infty}(\partial M)$ is to be understood in Theorem 1.3. The size of the neighbourhood of $\partial M$ in which the representation (2.17) holds is unimportant. Also, if for a given $f$ there exists such a representation with a particular defining function, a similar representation can be obtained with respect to any other defining function. In particular we can choose the defining function to coincide on some Gauss domain with the function $x$.

Definition 2.5. Under the assumptions of Definition 2.4 we say that a function $f$ on $\widetilde{M}$ is polyhomogeneous if for some real number $a$ it holds that $f \in \mathscr{A}_{p g h}^{a} \equiv \bigcap_{\alpha>a} W_{\alpha, b}^{a}$. In this case $f$ has an asymptotic expansion at $\partial M$ in the sense of (2.17) for all $N$ in $\mathbb{N}$, which by a common abuse of notation we write as the equality,

$$
f=\sum_{i=0}^{\infty} \sum_{j=0}^{N_{i}} f_{i j}(\log \varrho)^{j} \varrho^{s_{\imath}}
$$


with $N_{\imath}, s_{\imath}, f_{i j}$ as in Definition 2.4 (cf. [13]). We denote by $\mathscr{f}_{p h g}^{a, c}$ the space of $f \in \mathscr{C}_{\text {phg }}^{a}$ such that $f$ has an expansion of the form (2.18) with $N_{i}=0$ for all $i$ such that $s_{i}<c$.

The following result which spells out in particular the tangential regularity of the polyhomogeneous functions is immediate from the definitions.

Proposition 2.2 (Mapping properties). 1. Let $A \in \mathscr{O} \mathscr{P}_{b}^{m, \beta}$ and let $s \geq m$. Then $A$ defines continuous mappings

$$
A: C_{\delta, b}^{s, \alpha} \rightarrow C_{\delta+\beta, b}^{s-m, \alpha} \quad \text { and } \quad A: C_{\delta}^{s, \alpha} \rightarrow C_{\delta-m+\beta}^{s-m, \alpha}
$$

2. Under the same assumptions $A$ defines mappings

$$
A: W_{\alpha, b}^{a} \rightarrow W_{\alpha+\beta, b}^{a+\beta} \text { and } A: \mathscr{A}_{p h g}^{a} \rightarrow \mathscr{A}_{p h g}^{a+\beta}
$$

3. Let $A \in \mathscr{Q} \mathscr{P}_{0}^{m}$ and let $s \geq m$. Then $A$ defines a continuous mapping

$$
A: C_{\delta}^{s, \alpha} \rightarrow C_{\delta}^{s-m, \alpha}
$$

Proposition 2.3 (Commutation Property). Suppose we have operators $A \in \mathscr{Q} \mathscr{P}_{0}^{m}$ and $B \in \mathscr{Q} \mathscr{P}_{b}^{p}$ with $m, p \geq 1$. Then their commutator $[A, B]=A B-B A$ satisfies

$$
[A, B]=\sum_{\lambda \in I} C_{\lambda} D_{\lambda}
$$

where $I$ is a finite set, $C_{\lambda} \in \mathscr{O} \mathscr{P}_{0}^{m}$, and either $D_{\lambda} \in \mathscr{O} \mathscr{P}_{b}^{p-1}$ or $D_{\lambda}=1$, the identity operator (which is always the case if $p=1$ ).

Proof. The general case is readily reduced to the case where $A=A_{m} \ldots A_{2} A_{1}$ with $A_{i} \in \mathscr{Q} \mathscr{P}_{0}^{1}$ and $B=B_{p} \ldots B_{2} B_{1}$ with $B_{j} \in \mathscr{O}_{b}^{1}$. The case $p=1$ follows by induction on $m$ : It is obvious geometrically and easily checked analytically that $[A, B] \in \mathscr{Q} \mathscr{P}_{0}^{1}$ if $A \in \mathscr{O}_{0}^{1}$ and $B \in \mathscr{Q} \mathscr{P}_{b}^{1}$. Since

$$
\left[A_{m+1} A_{m} \ldots A_{1}, B\right]=A_{m+1}\left[A_{m} \ldots A_{1}, B\right]+\left[A_{m+1}, B\right] A_{m} \ldots A_{1}
$$

the result follows immediately for all $m \geq 1$. The case of arbitrary $m, p \geq 1$ follows now by induction on $p$ : having shown it already for $p=1$ and using the identity

$$
\left[A, B_{p+1} \ldots B_{1}\right]=\left[A, B_{p+1}\right] B_{p} \ldots B_{1}+B_{p+1}\left[A, B_{p} \ldots B_{1}\right]
$$

the result follows from the induction hypothesis. 


\section{Existence and Uniqueness of Solutions}

The proof of the existence and the uniqueness of the solution to (1.8), (1.9) is given by a series of lemmas.

Lemma 3.1. Let $(M, g, h, \varrho)$ be given as in the Definition 2.1 of conformally compact manifolds, with $\varrho$ chosen such that that $|d \varrho|_{h}=1$ on $\partial M$. Suppose that $w$ is a smooth positive solution on $\widetilde{M}$ of

$$
L_{h} w+n(n-1) w^{(n+2) /(n-2)}=0 \quad \text { with } \quad w \rightarrow \infty \quad \text { at } \partial M
$$

Then

$$
\liminf _{p \rightarrow \partial M}\left(w \varrho^{(n-2) / 2}\right)(p) \geq 1
$$

Proof. Because of the conformal covariance of the equation satisfied by $w$ we may assume without loss of generality that the Ricci scalar $R(h)$ is positive near $\partial M$. In case this condition were not satisfied we could replace $h$ and $\varrho$ by $\Theta^{2} h$ and $\Theta \varrho$ respectively, where the smooth function $\Theta>0$ is chosen as follows. Fix Gauss coordinates as described in Sect. 2 and set $\Theta=a x^{2}+1$ near $\partial M$. It follows from the transformation law (2.3) that we can determine the real number $a$ such that $R\left(\Theta^{2} h\right)>0$ near $\partial M$.

Consider now Gauss coordinates defined on a Gauss domain $U$ on which $0 \leq x<x_{0}$ for some positive $x_{0}$ and $R(h) \geq 0$. We may assume that $\varrho=x$ on $U$. Choose numbers $C, \varepsilon, \varepsilon_{1}, x_{1}>0$ with $C<1, x_{1}<x_{0}, \varepsilon \leq \varepsilon_{1}$, $x_{1}+\varepsilon_{1}<x_{0}$, and consider on the subset $W$ of $U$ where $0<x \leq x_{1}$ the function $v=C\left\{(x+\varepsilon)^{-(n-2) / 2}-\left(x_{1}+\varepsilon\right)^{-(n-2) / 2}\right\}$. The calculation shows that we can fix $\varepsilon_{1}, x_{1}$ suitable small such that

$L_{h} v+n(n-1) v^{(n+2) /(n-2)}=C v^{(n+2) /(n-2)}\left(n(n-1)\left(C^{4 /(n-2)}-1\right)+O(x+\varepsilon)\right) \leq 0$

on $W$ for all values of $C, \varepsilon$ in the range allowed above. Furthermore, we have $w>v$ near the boundary of $W$. It follows now from the maximum principle, see [9] and [3], that $v \leq w$ on $W$. Multiplying this inequality by $\varrho^{(n-2) / 2}$ and considering first the limit $\varepsilon \rightarrow 0$ and then the limit $C \rightarrow 1$ gives the result.

Lemma 3.2. Under the same assumptions on the function $w$ as in Lemma 3.1 there is a constant $C>0$ such that

$$
\limsup _{p \rightarrow \partial M}\left(w \varrho^{(n-2) / 2}\right)(p) \leq C
$$

Proof. Let again $U$ be a Gauss domain and $x$ the corresponding coordinate and $\varrho=x$ on $U$. Assume that the sequence of points $p_{k} \in \widetilde{M} \cap U$ is such that

$$
p_{k} \rightarrow p \in \partial M \quad \text { and } \quad\left(w \varrho^{(n-2) / 2}\right)\left(p_{k}\right) \rightarrow \limsup _{q \rightarrow \partial M}\left(w \varrho^{(n-2) / 2}\right)(q)
$$


and set $R_{k}=\frac{1}{3} \varrho\left(p_{k}\right)$. In the following we assume that $k \geq k_{0}$ with $k_{0}$ chosen large enough such that the points $p_{k}$ as well as the open balls $B_{3 R_{k}}\left(p_{k}\right)$ are contained in a convex normal neighbourhood of the point $p$ (in some smooth extension of $(M, h)$ ) and that $B_{3 R_{k}}\left(p_{k}\right) \subset \widetilde{M}$. If $\phi_{k} \in C_{0}^{\infty}\left(B_{3 R_{k}}\left(p_{k}\right)\right)$ with $\phi_{k}=1$ on $B_{2 R_{k}}\left(p_{k}\right)$ then, as has been shown in [4], there is a constant $C$ independent of $k$, such that

$$
\sup _{q \in B_{R_{k}}\left(p_{k}\right)} w(q) \leq C R_{k}^{-(n-2) / 2}\left(\int\left|\nabla \phi_{k}\right|_{h}^{n} d \mu(h)+\int \phi_{k}^{n} d \mu(h)\right)^{(n-2) / 2 n}
$$

where the integration is performed over $B_{3 R_{k}}\left(p_{k}\right)$. We choose $\phi_{k}$ as follows. Let $\psi \in C^{\infty}(\mathbb{R})$ be such that $\psi(t)=1$ for $t \leq 2, \psi(t)=0$ for $t \geq \frac{5}{2}$. Set $\phi_{k}(q)=\psi\left(\frac{1}{R_{k}} d\left(q, p_{k}\right)\right)$. Then it follows that the second integral on the right of Eq. (3.1) goes to zero as $k \rightarrow \infty$ while the first integral approach in this limit the values which one would get if one would replace $h$ by a flat metric. The result follows now from the fact that in the flat case the first integral is independent of $k$.

Lemma 3.3. Suppose the function $w$ satisfies the same condition as in Lemma 3.1. Assume $v$ is a smooth positive solution on $\widetilde{M}$ of $L_{h} v+n(n-1) v^{(n+2) /(n-2)}=0$ such that $\lim _{p \rightarrow \partial M}\left(v \varrho^{(n-2) / 2}\right)(p)=1$.

If either

$$
\lim _{p \rightarrow \partial M}\left(w \varrho^{(n-2) / 2}\right)(p)=1
$$

or

$$
\varrho \operatorname{grad}_{h}\left(v \varrho^{(n-2) / 2}\right) \text { is bounded on } \widetilde{M}
$$

then $w=v$.

Proof. Set $\tilde{h}=v^{4 /(n-2)} h$. Then by Eqs. (2.5) and (2.6) the positive smooth function $u=v^{-1} w$ on $\widetilde{M}$ satisfies

$$
L_{\tilde{h}} u=-n(n-1) u^{(n+2) /(n-2)}=R(\tilde{h}) u^{(n+2) /(n-2)}
$$

and by Lemmas 3.1, 3.2 we have

$$
1 \leq \liminf _{p \rightarrow \partial M} u(p) \leq \limsup _{p \rightarrow \partial M} u(p) \leq C
$$

for some constant $C \geq 1$ which can be set equal to 1 under our first hypothesis. It follows from the equation for $u$ above that the function $z=u-1$ satisfies an equation of the form

$$
\Delta_{\tilde{h}} z+g(u) z=0
$$

with a function $g \in C^{1}([0, \infty[)$ such that $g(0)=0, g(t)>0$ for $t>0$. We can now apply the strong maximum principle and the estimates (3.2) to conclude that $0 \leq z \leq C-1$ which gives our result under the first hypothesis. In the second 
case the result follows from Lemma 3.2 and the generalized maximum principle as described, e.g. in the article [8].

Theorem 3.4. Let $(M, g, h, \varrho)$ be given as in the Definition 2.1 of conformally compact manifolds with @ chosen that the condition (2.8) is satisfied. Then there exists a solution $u$ to the boundary value problem (1.8), (1.9) such that $z=u-1 \in C_{\delta}^{\infty}(M)$ for $\delta<n$.

Remark 3.1. Lemma 3.3 implies that this solution to the problem (1.8), (1.9) is unique. In Sect. 4 will be seen that the solution $u$ is of class $C^{n-1}$. This together with Lemma 3.3 and Theorem 3.4 implies Theorem 1.2.

Proof. Assume that $h$ has been scaled such that besides (2.8) also (2.9) is satisfied on some Gauss domain $U$. Set $w=1+a \varrho^{\eta}$ with some constants $a$ and $1 \leq \eta<n$. Then the calculation shows that

$$
L_{g} w+n(n-1) w^{(n+2) /(n-2)}=4 \frac{n-1}{n-2} a(\eta+1)(n-\eta) \varrho^{\eta}+O\left(\varrho^{\min (\eta+1, n)}\right) .
$$

This and the fact that $R(h)$ is bounded on $M$ implies that for a fixed choice of $\eta$ satisfying the conditions above we can determine sufficiently large positive constants $\bar{a}, \underline{a}, b$ such that the functions

$$
\bar{w}=\min \left(1+\bar{a} \varrho^{\eta}, b\right)
$$

and

$$
\underline{w}=\min \left(1-\underline{a} \varrho^{\eta}, 0\right)
$$

are weak global upper and lower solutions to our problem. It follows from standard arguments that there exist a solution $u$ with $\underline{w} \leq u \leq \bar{w}$ to the boundary value problem (1.8), (1.9) such that $u>0$ (cf. [4]) and $z=u-1 \in C_{\eta}^{0}(M) \cap C^{\infty}(\widetilde{M})$. Lemma 3.3 implies that the solution is unique and independent of the choice of $\eta$. By elliptic regularity and scaling arguments (cf. e.g. [1]) we conclude from this that $z \in C_{\delta}^{\infty}$ for $\delta<n$.

This last property is the starting point for our investigation of the smoothness behaviour of the solution near $\partial M$.

\section{Behaviour of the Solution near the Boundary}

Using on a suitable Gauss domain $U$ a defining function $\varrho$ and a scaling of the metric $h$ as considered in Eqs. (2.8), (2.9), such that $\varrho=x$ and observing (2.7), (2.12) we find that the Yamabe equation (1.8), written as equation for the unknown $z=u-1$, takes on $U$ the form

$$
A_{x, n} z+B z=F(p, z)
$$

where we use the notation

$$
A_{x, \xi}=-x^{2} D_{x}^{2}+(n-2) x D_{x}+\xi(\xi-n+1)
$$

with $\xi=n$,

$$
B=x^{2}\left(\Delta_{k}+\operatorname{tr}(\lambda) D_{x}\right)
$$


and

$$
F(p, z)=\frac{n-2}{4(n-1)}\left(-R_{n} x^{n}-R_{n} x^{n} z-n(n-1) G(z)\right)
$$

with $G(z)=(1+z)^{(n+2) /(n-2)}-1-\frac{n+2}{n-2} z$. In the following we shall study how the properties of the various terms in Eq. (4.1) affect the behaviour of the solution $z$ near $\partial M$.

\subsection{Some ODE Results}

In Gauss coordinates the operator (4.2) involves only partial derivatives with respect to $x$. For fixed $\xi \geq n-1$ consider the equation

$$
A_{x, \xi} u=f
$$

where now $A_{x, \xi}$ is viewed as an ODE operator on $\mathbb{R}^{+}$, together with the boundary condition

$$
u(x) \rightarrow 0 \quad \text { as } \quad x \rightarrow 0_{+} .
$$

In the case $f=0$ Eq. (4.5) is Euler's equation. Its solution are given by

$$
u=u_{0} x^{\xi}+u_{-} x^{-\xi+n-1}
$$

with constants $u_{0}, u_{-}$. This allows to determine the solution of Eq. (4.5) for the more general right-hand side by standard methods. Let the kernel $H_{\xi}(x, \tilde{x})$ be given by

$$
H_{\xi}(x, \tilde{x})=C_{\xi}\left(-x^{\xi} \tilde{x}^{n-1-\xi} H(x-\tilde{x})+x^{n-1-\xi} \tilde{x}^{\xi} H(x-\tilde{x})\right)
$$

with $C_{\xi}=(2 \xi+1-n)^{-1}$, where $H(s)$ is the Heavyside step function. Then, for suitable given $f$, the function

$$
z(x)=\int H_{\xi}(x, \tilde{x}) f(\tilde{x}) \tilde{x}^{-n} d \tilde{x}
$$

solves $A_{x, \xi} u=f$.

Since we are interested only in the behaviour of the solutions near $x=0$ we consider spaces of functions on $I=] 0,1$ [ which reflect the radial behaviour of the functions considered in Definitions 2.2 and 2.5. We use obvious analogues to the norms (2.15) to define the spaces $C_{\alpha}^{s}(I)$. We denote by $\mathscr{C}_{p h g}^{a}(I)$ the functions $\left.\left.f \in C^{\infty}(] 0,1\right]\right)$ which have at $x=0$ an asymptotic expansion of the form (2.18) with $\varrho=x$ and some constants $f_{i j}$. Finally we set $W_{\alpha}^{a}(I)=\mathscr{A}_{p h g}^{a}(I)+C_{\alpha}^{\infty}(I)$.

Lemma 4.1. Let $\xi \geq n-1, \alpha>\xi$, suppose that $f=O\left(x^{\alpha}\right)$, define

$$
H_{\xi}[f](x)=C_{\xi} x^{\xi}\left(-\int_{0}^{x} \tilde{x}^{-1-\xi} f(\tilde{x}) d \tilde{x}+x^{n-1-2 \xi} \int_{0}^{x} \tilde{x}^{\xi-n} f(\tilde{x}) d \tilde{x}\right)
$$


Then $H_{\xi}[f]$ is the unique solution to (4.5), (4.6) which behaves as $O\left(x^{\alpha}\right)$ at $x=0$. Moreover the operator $H_{\xi}$ given by (4.9) defines a map

$$
H_{\xi}: C_{\alpha}^{\infty}(I) \rightarrow C_{\alpha}^{\infty}(I)
$$

and for $a>\alpha$ it restricts to mappings

$$
H_{\xi}: \mathscr{A}_{p h g}^{a}(I) \rightarrow \mathscr{A}_{p h g}^{a}(I), \quad H_{\xi}: W_{\alpha}^{a}(I) \rightarrow W_{\alpha}^{a}(I)
$$

Proof. For functions $f$ satisfying $f=O\left(x^{\alpha}\right)$ with $\alpha>\beta$ the integral

$$
u_{0}=\int_{0}^{x_{1}} \tilde{x}^{-1-\beta} f(\tilde{x})
$$

exists, and the results follow by straightforward estimations and/or integration.

\subsection{The Scalar Laplace-Beltrami Operator}

We generalize slightly the right-hand side of Eq. (4.1) and study now on $U \simeq$ $\left[0, x_{0}\left[\times \partial M\right.\right.$, where $x_{0}>0$, the operator

$$
A_{\xi}=\Delta_{g}+\xi(\xi-n+1)=A_{x, \xi}+B
$$

where the operators on the right-hand side are given on $U$ by (4.2) and (4.3) respectively. The interval $I$ in Lemma 4.1 can obviously be replaced by the interval $\left[0, x_{0}\right.$ [ without changing any of the results in an essential way. Since the operator $B$ is in $\mathscr{P}_{b}^{2,1}$ it defines by Proposition 2.2 a continuous mapping $B: C_{\delta, b}^{\infty} \rightarrow C_{\delta+1, b}^{\infty}$. This means that with respect to the radial regularity we can treat $B$ as a lower order perturbation of $A_{x, \xi}$. Basic for our discussion are the following two lemmas:

Lemma 4.2 (Isomorphism property). Let $s \geq 0$; for $\xi \geq n-1$ consider the operator

$$
A_{\xi}=\Delta_{g}+\xi(\xi-n+1) \text {. }
$$

1. For $0<\alpha<1$ and for all $\delta$ such that

the map

$$
0<\delta<\xi
$$

$$
A_{\xi}: C_{\delta}^{s+2, \alpha}(M) \rightarrow C_{\delta}^{s, \alpha}(M)
$$

is an isomorphism.

2. For $0<\alpha<1$ and for all $\delta$ such that

$$
n-1-\xi<\delta<\xi
$$


there exists $x_{*}, 0<x_{*}<x_{0}$ (depending only upon $n, h_{i j}, \xi$ and $\delta$ ) such that for every $f_{*} \in C^{s+2, \alpha}\left(N_{x_{*}}\right)$ the map

$$
A_{\xi}:\left\{f \in C_{\delta}^{s+2, \alpha}\left(\widetilde{M}_{x_{*}}\right) \mid f\left(x_{*}, \cdot\right)=f_{*}(\cdot)\right\} \rightarrow C_{\delta}^{s, \alpha}\left(\widetilde{M}_{x_{*}}\right)
$$

where $\widetilde{M}_{x_{*}}=\left\{p \in M: 0<x(p) \leq x_{*}\right\}$, is an isomorphism.

Proof. Point 1) can be shown be standard techniques (cf. e.g. [8]) using the weak barriers given by $\phi_{ \pm}= \pm \min \left(1, C x^{\delta}\right)$, with a constant $C$ large enough. Point 2) follows from the fact that the functions $\phi_{ \pm}= \pm C x^{\delta}$ are barriers for $x \leq x_{0}\left(h_{\imath \jmath}, n, \xi, \delta\right.$ ) (for $\delta \leq 0$ one should use the generalized maximum principle, cf. [8]).

For our applications the importance of Lemma 4.2 stems from the following:

Lemma 4.3. Let $\xi \geq n-1, s \geq 0,0<\alpha<1$, suppose that

$$
\begin{gathered}
A_{\xi} u=f, \quad f \in C_{\delta}^{s, \alpha}, \quad n-1-\xi<\delta<\xi, \\
u \in C^{2}(\widetilde{M}) \cap C_{\sigma}^{o, \alpha}, \quad \sigma>n-1-\xi .
\end{gathered}
$$

Then

$$
u \in C_{\delta}^{s+2, \alpha}
$$

Here $C^{2}(\widetilde{M})$ only indicates local differentiability, no norm is involved.

Proof. By elliptic regularity and scaling arguments (cf. e.g. [1]) we have $u \in C_{\sigma}^{s+2, \alpha}$. Let $x_{0}(\delta)$ and $x_{0}(\sigma)$ be given by Lemma 4.2, point 2), set $x_{0}=\min \left(x_{0}(\delta), x_{0}(\sigma)\right)$, define $u_{0}=\left.u\right|_{M_{x_{0}}}$. If $\sigma>\delta$ there is nothing to prove, suppose thus $\delta>\sigma$. By Lemma 4.2, point 2) there exists $\tilde{u} \in C_{\delta}^{s+2, \alpha}\left(I_{x_{0}}\right)$ (recall that $I_{x_{0}}=\left\{p \in M: 0<x(p) \leq x_{0}\right\}$ ) satisfying $\left.\tilde{u}\right|_{M_{x_{0}}}=u_{0}, A_{\xi} \tilde{u}=f$. Since $\sigma<\delta$ we have $\tilde{u} \in C_{\sigma}^{s+2, \alpha}$, by injectivity of $A_{\xi}$ on $\left\{u \in C_{\delta}^{s+2, \alpha}\left(I_{x_{0}}\right): u_{0}=\left.u\right|_{M_{x_{0}}}\right\}$ it follows that $\left.u\right|_{I_{x_{0}}}=\tilde{u}$, thus $u \in C_{\delta}^{s+2, \alpha}(M)$.

Proposition 4.4 ( $\mathscr{b}$ regularity). Suppose that for some integer $m \geq 1$, all integers $s \geq 0$ and for some $0<\alpha<1$ the operator $A \in \mathscr{G} \mathscr{P}_{0}^{m}$ is an isomorphism $A: C_{\delta}^{s+m, \alpha} \rightarrow C_{\delta}^{s, \alpha}$ for all $\left.\delta \in I=\right] c_{0}, c_{1}$ [, where $c_{1}>c_{0}+1$. Fix a $\delta, \delta^{\prime} \in I$ such that $\delta^{\prime}>\delta$ and $\delta^{\prime}-c_{0}>1$. If $u \in C_{\delta}^{m, \alpha}$ satisfies the equation

$$
A u=f
$$

for some $f \in C_{\delta^{\prime}, b}^{s, \alpha}$, then in fact $u \in C_{\delta^{\prime}, b}^{s, \alpha}$. In particular, if $f \in C_{\delta^{\prime}, b}^{\infty}$ then $u \in C_{\delta^{\prime}, b}^{\infty}$. Proof. From the isomorphism property ensues that $u \in C_{\delta^{\prime}}^{s+m, \alpha}$. If $B \in \mathscr{Q}_{b}^{1}$ we get from Proposition 2.2 that $B u \in C_{\delta^{\prime}-1}^{s+m-1, \alpha}$. But by Proposition 2.3 and Proposition 2.2, we obtain

$$
A(B u)=[A, B] u+B f \in C_{\delta^{\prime}}^{s-1, \alpha}
$$


whence, since $A$ is an isomorphism for the scale of spaces, we get in fact $B u \in$ $C_{\delta^{\prime}}^{s+m-1, \alpha}$. We can now apply this argument inductively to prove that $u \in C_{\delta^{\prime}, b}^{s+m-1, \alpha}$.

The following result provides radial regularity for solutions of equations of the form

$$
\left(A_{x, \xi}+B\right) u=f
$$

for $f$ and $u$ with $\mathscr{T}_{b}$ regularity. This result is well known, ${ }^{1}$ we include the proof that follows here because it is much simpler than the usual Mellin transform approach.

Lemma 4.5. 1. Assume that $f \in C_{\alpha, b}^{\infty}(M)$ for $\alpha>\xi \geq n-1$. Suppose that $u \in C_{\xi-\varepsilon, b}^{\infty}(M)$ with $0 \leq \varepsilon<1$ satisfies (4.11) on $U$. Then $u$ is of the form $u=x^{\xi}\left(\sum_{i=0}^{[\alpha-\xi]} u_{i} x^{2}+u_{*}\right)$, where $u_{i} \in C^{\infty}(\partial M)$ and $u_{*} \in C_{\alpha-\xi, b}^{\infty}$, in particular $u \in W_{\alpha, b}^{\xi}(M)$.

2. Assume $a \geq 1,0 \leq \varepsilon<1$ and let $u \in C_{a-\varepsilon, b}^{\infty}$ satisfy Eq. (4.11) on some neighbourhood of $\partial M$. If $f \in W_{\alpha, b}^{a}$ with $\alpha>\xi \geq n-1$ then $u \in W_{\alpha, b}^{\min (a, \xi)}$. In particular, if $u$ satisfies the same conditions as above and $f \in \mathscr{A}_{p h g}^{a}$ for some $a \geq 1$ then $u \in \mathscr{A}_{p h g}^{\min (a, \xi)}$.

Proof. 1. Since $B \in \mathscr{O} \mathscr{P}_{b}^{2,1}$ it follows from Proposition 2.2 that

$$
A_{x, \xi} u=f-B u \in C_{\alpha^{\prime}, b}^{\infty}
$$

where $a^{\prime}=\min (\xi-\varepsilon+1, \alpha)>\xi$. On a fixed integral curve of the vector field $\operatorname{grad}_{h} x$ on $U$ it follows from the ODE (4.12) and from $u=o\left(x^{n-1-\xi}\right)$ that $u=x^{\xi} u_{0}+H_{\xi}[f-B u]$. Here $u_{0}$ is constant on the integral curves but must be considered as a function on $\partial M$. It follows from Lemma 4.1 that $H_{\xi}[f-B u] \in C_{\alpha^{\prime}}^{\infty}$. From the expression for $H_{\xi}$ given in (4.9) it follows that in fact $H_{\xi}[f-B u] \in C_{\alpha^{\prime}, b}^{\infty}$. Using the smoothness assumptions on $u$ we conclude that $u_{0} \in C^{\infty}(\partial M)$. If $\alpha^{\prime}<\alpha$ we can repeat the procedure. Assume that $u$ has been shown to have an expansion of the form

$$
u=x^{\xi}\left(\sum_{i=0}^{l} v_{i} x^{i}+v_{*}\right)
$$

with $v_{i} \in C^{\infty}(\partial M), v_{*} \in C_{\alpha-\xi, b}^{\infty}$, and $l<[\alpha-\xi]$. Then we may again use Lemma 4.1 to conclude that

$$
u=x^{\xi} u_{0}^{\prime}+\sum_{i=0}^{l} v_{i} H_{\xi}\left[B\left(x^{\imath+\xi}\right)\right]+H_{\xi}\left[f-B\left(x^{\xi} v_{*}\right)\right]
$$

and it is easily seen that this pushes the expansion one step further while preserving all the required smoothness properties.

\footnotetext{
${ }^{1}$ We are grateful to G. Lysik and B. Ziemian for pointing out to us the Mellin transform approach to this problem
} 
2. Let

$$
f=\sum_{i=0}^{I} \sum_{j=0}^{N_{i}} f_{i, j} x^{s_{i}} \log ^{j} x+r, \quad r \in C_{\alpha, b}^{\infty} .
$$

It is easily seen by induction that there exist functions $v_{i, j} \in C^{\infty}(\partial M), i=1, \ldots, \tilde{I}$, $j=0, \ldots, N_{i}+1$, for some $\tilde{I}$, such that

$$
A_{\xi}\left(\sum_{i=0}^{\tilde{I}} \sum_{j=0}^{N_{\imath}+1} v_{i, j} x^{\tilde{s}_{\imath}} \log ^{j} x\right)=\sum_{i=0}^{I} \sum_{j=0}^{N_{i}} f_{i, j} x^{s_{\imath}} \log ^{j} x+\tilde{r}
$$

where $\left\{\tilde{s}^{i}\right\} \equiv\left\{s_{\imath}+k: k \in \mathbb{N} \cup\{0\}, s_{i}+k \leq \alpha+1\right\}$, for some $\tilde{r} \in \mathscr{A}_{p h g}^{\alpha+1} \subset$ $C_{\alpha+1-\varepsilon, b}^{\infty} \subset C_{\xi-\varepsilon}^{\infty}$, for any $\varepsilon>0$. By Lemma 4.2 there exists $\tilde{u} \in C_{\xi-\varepsilon}^{\infty}$ such that $A_{\xi} \tilde{u}=r-\tilde{r}$, by Lemma 4.2 and Proposition 4.4 we have $\tilde{u} \in C_{\xi-\varepsilon, b}^{\infty}$. Since both $u$ and $\tilde{u}+\sum_{i=0}^{\tilde{I}} \sum_{j=0}^{N_{\imath}+1} v_{i, j} x^{\tilde{s}_{\imath}} \log ^{j} x$ are in $C_{a-\varepsilon}^{\infty}$, the uniqueness part of Lemma 4.2, point 1, shows that $u=\tilde{u}+\sum_{i=0}^{\tilde{I}} \sum_{j=0}^{N_{i}+1} v_{i, j} x^{\tilde{s}_{\imath}} \log ^{j} x$, and since $r-\tilde{r} \in C_{\alpha, b}^{\infty}$ we have $\tilde{u} \in W_{\alpha, b}^{\xi}$ by point 1 of this Lemma.

We next consider the regularity of solutions on a conformally compact manifold $M$ to equations of the type $A_{\xi} u=f$, under various conditions on $f$ :

Theorem 4.6 (Regularity). Let $\xi>n-1$ and assume that $u \in C_{\delta}^{2}$, with some $\delta>n-\xi$, is a solution to the equation

$$
A_{\xi} u=f
$$

1. If $f \in C_{\alpha, b}^{\infty}(M)$ for some $\alpha>\xi$, then $u$ is of the form $u=x^{\xi}\left(\sum_{i=0}^{[\alpha-\xi]} u_{i} x^{i}+u_{*}\right)$, where $u_{i} \in C^{\infty}(\partial M)$ and $u_{*} \in C_{\alpha-\xi, b}^{\infty}$.

2. Assume ${ }^{2}$ that $a>n-\xi$. If $f \in W_{\alpha, b}^{a}$ with $\alpha>\xi$, then $u \in W_{\alpha, b}^{\min (\xi, a)}$. In particular, if $f \in \mathscr{A}_{p h g}^{a}$, then $u \in \mathscr{A}_{p h g}^{\min (\xi, a)}$. If $f \in \mathscr{A}_{p h g}^{\xi+\varepsilon, \ell}$ for some $\ell>\xi+\varepsilon$, then $u$ has no log-terms until the order $\ell$.

3. Assume that $\xi$ is an integer and that $f \in C^{\infty}(M)$. Then there exists $u_{1}, u_{2} \in$ $C^{\infty}(M)$, with $u_{2}=O\left(x^{\xi}\right)$, such that

$$
u=u_{1}+u_{2} \log x
$$

If $\left.f\right|_{\partial M}=0$, then $\left.u\right|_{\partial M}=0$. Further, if in addition $f=o\left(x^{\xi}\right)$, then $u \in C^{\infty}(M)$. Proof. By Lemma 4.2 and Proposition $4.4 u$ in tangentially smooth, and point 1 together with the first part of point 2 follow from Lemma 4.5. The second part of point 2 follows by comparing the coefficients in an asymptotic expansion of $A_{\xi} u$ with those of $f$. To prove point 3 , note that by point $2 u$ is polyhomogeneous and again

2 The hypotheses $a, \delta>n-\xi$ can be relaxed to $a, \delta>n-\xi-1$ by a difference quotient argument (which is sharp), cf. [2]; this will however not be needed in this paper 
comparing the coefficients in an asymptotic expansion of $A_{\xi} u$ with those of $f$ one finds that $u$ has an asymptotic expansion of the form

$$
u=\sum_{i=0}^{\infty} u_{i, 1} x^{\imath}+\log x \sum_{i=0}^{\infty} u_{i, 2} x^{\xi+i}
$$

By Borel's lemma there exist functions $\tilde{u}_{1}, u_{2} \in C^{\infty}(M)$ with the asymptotic expansions

$$
\tilde{u}_{1}=\sum_{i=0}^{\infty} u_{i, 1} x^{i}, \quad u_{2}=\sum_{i=0}^{\infty} u_{i, 2} x^{\xi+\imath},
$$

so that $\tilde{u} \equiv u-\tilde{u}_{1}-u_{2} \log x$ is polyhomogeneous, vanishes to arbitrary order at $\partial M$ together with all derivatives, and thus $\tilde{u} \in C_{\infty}(M)$. It follows that $L \tilde{u} \in C^{\infty}(M)$, and $L \tilde{u}$ vanishes to arbitrary order at $\partial M$, so that point 1 implies $\tilde{u} \in C^{\infty}(M)$. Setting $u_{1}=\tilde{u}+\tilde{u}_{1}$ we have $u=u_{1}+u_{2} \log x$, and the result follows.

\subsection{Radial Regularity for the Nonlinear Case and Proof of Theorem 1.3}

In this section we consider solutions to an equation of the form

$$
A_{\xi} w=F(w) \equiv U w+V+G(w)
$$

where $U, V \in \mathscr{b}_{p h g}^{1}$ and $G \in C^{\infty}(\mathbb{R})$, with $G(0)=G^{\prime}(0)=0$. First we prove that a solution which decays sufficiently rapidly at $\partial M$ must be tangentially smooth.

Lemma 4.7. Let $\xi>n-1, \delta>\max (0, n-\xi)$, assume that $w \in C_{\delta}^{\infty}$ is a solution of (4.14). Then for all $a<\xi$ we have in fact $w \in W_{\alpha, b}^{1}$, i.e. $w$ is tangentially smooth.

Proof. Suppose that $\delta<1 / 2$, then $F(w) \in C_{2 \delta}^{\infty}$, by Lemma 4.2 it follows that $w \in C_{2 \delta}^{\infty}$, and repeating this argument a finite number of times if necessary we get $w \in C_{\delta}^{\infty}, \delta>1 / 2$. It then follows

$$
F(w)=F_{\eta}+R_{\eta} \equiv \sum_{i=1}^{k} \sum_{j=0}^{N_{\imath}} F_{i, j} x^{s_{\imath}} \log ^{\jmath} x+R_{\eta}
$$

with $R_{\eta} C_{\eta}^{\infty}, \eta \geq \min (2 \delta, 1+\delta), s_{k} \geq \eta$, and $F_{i, j} \in C^{\infty}(\partial M)$. Lemma 4.2 and Theorem 4.6 show that we can find $w_{\eta} \in \mathscr{A}_{p h g}^{1}, r_{\eta} \in C_{\eta}^{\infty}$ if $\eta<\xi$ or $r_{\eta} \in C_{\alpha}^{\infty}$ for any $\alpha<\xi$ if $\eta \geq \xi$, such that

$$
A_{\xi} w_{\eta}=F_{\eta}, \quad A_{\xi} r_{\eta}=R_{\eta}
$$

Uniqueness implies $w=w_{\eta}+r_{\eta}$, and repeating the above argument a finite number of times one obtains $\eta \geq \alpha$, thus $w=w_{\alpha}+r_{\alpha}, w_{\alpha} \in \mathscr{A}_{\text {phg }}^{1}, r_{\alpha} \in C_{\alpha}^{\infty}$. Let now $B=\mathcal{Q}_{b}^{1}$, we have

$$
A_{\xi}(B w)=\left[A_{\xi}, B\right] w-B F(w) \in W_{\alpha}^{1},
$$


and since $B w=B w_{\alpha}+B r_{\alpha}, B w_{\alpha} \in \mathscr{A}_{p h g}^{1}, B r_{\alpha} \in C_{\alpha-1}^{\infty}, \alpha-1>0$, one concludes from Lemma 4.2 that $B w \in W_{\alpha}^{1}$. An induction argument shows that for every $B_{\imath} \in \mathscr{O P}_{b}^{1}, i=1, \ldots, N$, we have $B_{1} \cdots B_{i} w \in W_{\alpha}^{1}$, thus $w \in W_{\alpha, b}^{1}$, which had to be established.

This result enables us to prove polyhomogeneity.

Theorem 4.8. Under the assumptions of Lemma 4.7, we have $w \in \mathscr{C}_{p h g}^{1}$.

Proof. By Lemma 4.7, $w \in W_{\eta, b}^{1}$ for some $\eta>1$. It follows that

$$
A_{\xi} w=U w+V+G(w) \in W_{\eta^{\prime}, b}^{1} \quad \text { with any } \quad \eta^{\prime}<\eta+1 .
$$

Applying Theorem 4.6 inductively now shows that $w \in W_{\alpha, b}^{1}$ for any $\alpha$ and hence $w \in \mathscr{A}_{p h g}^{1}$.

If the term $V$ satisfies an additional requirement, then we can prove that the solution is actually smooth.

Corollary 4.9. If $U, V \in C^{\infty}(M), U=O(x), V=O\left(x^{\xi+1}\right)$, then $x^{-\xi} w \in C^{\infty}(M)$. In particular, if $\xi$ is an integer, then $w \in C^{\infty}(M)$.

Proof. By Theorem 4.8, $w \in \mathscr{A}_{p h g}^{1}$, and hence after iterating

$$
A_{\xi} w=U w+V+G(w) .
$$

Using Theorem 4.6 part (2) we find that $w \in \mathscr{A}_{p h g}^{\xi, 2 \xi}$. Iterating this leads to $w \in \mathscr{C}_{p h g}^{\xi, \ell}$ for any $\ell$ and hence the corollary is proved.

From the results above we can deduce Theorem 1.3. We know already that the Yamabe equation can be written in the form (4.1) with (4.2), (4.3), (4.4) for an unknown $z$, which by Theorem 3.4 is in $C_{\delta}^{\infty}$ for $\delta<n$. Since the function $G$ on the right-hand side of (4.4) is smooth in a domain strictly larger than the set $\{z>-1\}$, where $z$ takes its values, we can assume $G$ to be extended suitable to a function that satisfies the requirements on the function $G$ considered above. Now parts 1 and 2 of Theorem 1.3 follow from Lemma 2.1, Theorem 4.8, and Corollary 4.9. Part 3 will follow from parts 1 and 2 and Lemma 5.2, which will be given in the next section.

\section{Smoothness of the Hyperboloidal Initial Data}

We shall in the following discuss a number of equivalent conditions under which the solution $u$ of the boundary value problem (1.8), (1.9) extends smoothly to $\partial M$. These will allow us to prove part 3 of Theorem 1.3 and Theorem 1.1.

We assume that on some Gauss domain $U$ the condition (2.8) and (2.9) are satisfied. Then we have

$$
R_{n} x^{n}=x^{2} R(h)-2(n-1) x \operatorname{tr}(\lambda) \quad \text { on } \quad U .
$$

Taking $x$-derivatives of (5.1) and evaluating on $\partial M$ we get

$$
D_{x}^{k} \operatorname{tr}(\lambda)=\frac{k}{2(n-1)} D_{x}^{k-1} R(h) \quad \text { on } \quad \partial M \quad \text { for } k=0, \ldots, n-2
$$


and the equivalent "regularity conditions"

$$
\left.R_{n}\right|_{\partial M}=0 \Leftrightarrow 0=D_{x}^{n-1} \operatorname{tr}(\lambda)-\frac{1}{2} D_{x}^{n-2} R(h) \quad \text { on } \quad \partial M .
$$

One would like to give the regularity condition in terms of well understood quantities on $\partial M$ which do no involve non-tangential derivatives. This is more desirable since the quantities entering the expression above depend very much on our choice of scaling. As we have seen in the proof of Lemma 2.1 there is still some freedom in the scaling of $h$. Changing this scaling will in general also change the family of hypersurfaces $M_{s}$ in $U$ and the second fundamental forms obtained for different scalings will be related to each other in a complicated way.

In the case of dimension $n=3$ we can derive a simple condition. First we introduce a useful function.

Lemma 5.1. Let $\lambda_{A B}^{*}$ be the traceless part of $\lambda_{A B}$, define on $\partial M$ the function

$$
C(h, \partial M, M)=\delta^{A} \delta^{B} \lambda_{A B}^{*}+\lambda^{* A B} R_{A B}-\frac{1}{2} \operatorname{tr}(\lambda) \lambda^{* A B} \lambda_{A B}^{*} .
$$

Then $C(h, \partial M, M)$ is a conformal density of weight -3, i.e. it satisfies $C\left(\Theta^{2} h, \partial M, M\right)=\Theta^{-3} C(h, \partial M, M)$ for all smooth positive functions $\Theta$ on $M$.

Proof. The proof of the transformation behaviour of $C$ under transitions $h \rightarrow \Theta^{2} h$ follows by a straightforward calculation from (2.2) and the transformation law $\lambda \rightarrow \Theta \lambda+k\langle n, d \Theta\rangle$ for the second fundamental form on $\partial M$, where $n$ denotes the inward pointing unit normal of $\partial M$ with respect to the metric $h$.

This allows us to give the regularity condition in the form

Lemma 5.2. In the case of dimension 3 the solution $u$ to the Yamabe problem (1.8), (1.9) is smooth on $M$ if and only if the conformal structure defined by the metric $h$ is such that $C(h, \partial M, M)=0$ on $\partial M$.

Proof. Using the same tetrad conventions as in Sect. 2.1, the embedding equations for the hypersurfaces $M_{s}$ into $M$ are given by Gauss' equation

$$
R_{A B C D}=r_{A B C D}+\lambda_{A D} \lambda_{B C}-\lambda_{A C} \lambda_{B D}
$$

and its contractions

$$
\begin{gathered}
R_{B D}-R_{1 B 1 D}=r_{B D}+\lambda_{C D} \lambda_{B}^{C}-\lambda_{C}^{C} \lambda_{B D} \\
R-2 R_{11}=r+\lambda_{C D} \lambda^{C D}-\left(\lambda_{C}^{C}\right)^{2}
\end{gathered}
$$

and by Codazzi's equation and its contraction

$$
\begin{aligned}
R_{1 B C D} & =\delta_{C} \lambda_{D B}-\delta_{D} \lambda_{C B} \\
R_{1 C} & =\delta_{C} \lambda_{D}^{D}-\delta_{D} \lambda_{C}^{D}
\end{aligned}
$$

Furthermore we have the equations

$$
\begin{aligned}
R_{1 A 1 B} & =e_{1}\left(\lambda_{A B}\right)-\lambda_{A C} \lambda_{B}^{C} \\
R_{11} & =e_{1}\left(\lambda_{A}^{A}\right)-\lambda_{A B} \lambda^{A B}
\end{aligned}
$$


The curvature quantities indicated by a capital $R$ on the left-hand side of these formulae are the Riemann tensor, the Ricci tensor, respectively the Ricci scalar defined by the metric $h$, while on the right-hand side are denoted by a small $r$ the corresponding quantities derived from the interior metric $k$ on the hypersurface $M_{s}$ in $U$. Contractions on the right-hand sides are performed with the metric $k$.

We can replace the expression on the right-hand side of (5.3) by a lower order expression in the following way. We take derivatives of (5.10) to obtain

$$
D_{x}^{n-1} \operatorname{tr}(\lambda)=D_{x}^{n-2} R_{11}+D_{x}^{n-2}\left(\lambda_{A B} \lambda^{A B}\right) .
$$

From the contracted Bianchi identity and (5.8) we derive

$$
\frac{1}{2} D_{x} R=D^{a} R_{a 1}=D_{x} R_{11}-\Delta_{k} \operatorname{tr}(\lambda)-\delta^{A} \delta^{B} \lambda_{A B}-\operatorname{tr}(\lambda) R_{11}+\lambda^{A B} R_{A B} .
$$

Taking derivatives of this equation and using the previous equation we arrive at

$$
\begin{aligned}
& D_{x}^{n-1} \operatorname{tr}(\lambda)-\frac{1}{2} D_{x}^{n-2} R(h) \\
& \quad=D_{x}^{n-2}\left(\lambda_{A B} \lambda^{A B}\right)+D_{x}^{n-3}\left(\Delta_{k} \operatorname{tr}(\lambda)+\delta^{A} \delta^{B} \lambda_{A B}+\operatorname{tr}(\lambda) R_{11}-\lambda^{A B} R_{A B}\right) .
\end{aligned}
$$

We assume now $n=3$, use Eqs. (5.9), (5.5) to obtain $D_{x} \lambda_{A B}=R_{A B}-r_{A B}+$ $\operatorname{tr}(\lambda) \lambda_{A B}$, contract this with $\lambda^{A B}$, and use the resulting expression to replace the first term on the right of the previous equation. We obtain

$$
\begin{aligned}
& D_{x}^{2} \operatorname{tr}(\lambda)-\frac{1}{2} D_{x} R(h) \\
& =-2 r \operatorname{tr}(\lambda)+2 \operatorname{tr}(\lambda) \lambda_{A B} \lambda^{A B}+\Delta_{k} \operatorname{tr}(\lambda)+\delta^{A} \delta^{B} \lambda_{A B}+\operatorname{tr}(\lambda) R_{11}-\lambda^{A B} R_{A B} .
\end{aligned}
$$

Observing now the scaling behaviour of $C$ and the fact that in our present scaling condition (2.11) is satisfied, we get our result from (5.3).

Proceeding in a similar way in the case of higher dimensions would give complicated terms, non-linear in the curvature of $h$.

The following example shows that the condition given in Lemma 5.2 is not trivial. Let $M$ be the solid torus embedded in $\mathbb{R}^{3}$ whose boundary is given by the 2 -surface

$$
\begin{aligned}
\partial M=\left\{(x, y, z) \in \mathbb{R}^{3} \mid x\right. & =(R+r \cos \theta) \cos \phi, y=(R+r \cos \theta) \sin \phi, \\
z & =r \sin \theta)\},
\end{aligned}
$$

where $0 \leq \theta, \phi<2 \pi$ and $r, R$ are given numbers satisfying $0<r<R$. Assume that $h$ is the metric induced on $M$ by flat standard metric on $\mathbb{R}^{3}$. Then we find

$$
C(h, \partial M, M)=\frac{1}{4} R\{r(R+r \cos \theta)\}^{-3}\left(R^{2}+2 r^{2}+4 R r \cos \theta\right)
$$

which vanishes only on sets of measure zero on $\partial M$. 


\subsection{Proof of Theorem 1.1}

Suppose $u$ is the solution to the boundary value problem (1.8), (1.9), set $\Omega=\varrho u^{-2}$ and $\tilde{g}=\Omega^{-2} h$. We shall study the behaviour of the tensor fields (1.3), (1.4), and (1.5) on a Gauss domain $U$ where we assume the conditions (2.8) and (2.9) to be satisfied. It follows then from Theorem 1.3 that $\Omega$ is near $\partial M$ of the form

$$
\Omega=x-2 u_{3,0} x^{4}-2 u_{3,1}(\log x) x^{4}+\text { higher order } .
$$

Thus $\Omega \in C^{3}(M)$.

The condition that $\Omega$ be smooth is equivalent to the condition that $u$ be smooth which imposes the condition $C=0$ on our "free data" $(M, h)$.

Using the above expression for $\Omega$ we can calculate from it the trace free part of the Hessian of $\Omega$ and get

$$
D_{a} D_{b} \Omega-\frac{1}{3} h_{a b} D_{c} D^{c} \Omega=-\lambda_{a b}+\frac{1}{3} h_{a b} \operatorname{tr}(\lambda)+O\left(x^{2} \log x\right),
$$

where $\lambda$ is considered as a tensorfield on $U$ which contracts to zero with the gradient of $x$. Thus the requirement that the field (1.4) extends smoothly to $\partial M$ implies in view of our assumption (2.11) the conformally invariant condition

$$
\lambda^{*}=0 \quad \text { on } \quad \partial M
$$

which implies in turn the smoothness of $\Omega$ by Lemma 5.2.

Assuming (5.12) to be satisfied and using Eqs. (5.2), (5.6), (5.9), and (5.10) we find that the field $s_{a b}$ extends smoothly to $\partial M$ and takes there the following values:

$$
\begin{gathered}
s_{1 A}=0, s_{11}=-\frac{1}{3} D_{x} \operatorname{tr}(\lambda)=-\frac{1}{12} R \\
s_{A B}=D_{x} \lambda_{A B}-\frac{1}{3} h_{A B} D_{x} \operatorname{tr}(\lambda)=R_{A B}-\frac{1}{3} R h_{A B} .
\end{gathered}
$$

Let $C_{a b}$ denote the electric part of the Weyl curvature (note that the magnetic part of the Weyl tensor vanishes because of our hypothesis $\left.\tilde{\chi}=\frac{1}{3} \operatorname{tr}(\tilde{\chi}) \tilde{g}\right)$; it is now straightforward to check that

$$
C_{a b}=\Omega d_{a b}=R_{a b}(h)-\frac{1}{3} R(h) h_{a b}-s_{a b}
$$

goes to zero at $\partial M$ which implies that $d_{a b}$ also extends smoothly to $\partial M$. Also note that from what has been said it follows that $C_{a b}$ goes to zero at $\partial M$ if and only if $s_{a b}$ is bounded which we have seen to be true if and only if (5.12) is satisfied. From this follows Theorem 1.1.

We end the article with a few remarks. Comparing (1.5) with (2.4) shows that

$$
C_{a b}=R_{a b}(\tilde{g})-\frac{1}{3} \tilde{g}_{a b} R(\tilde{g}) .
$$

Beside the implication of (5.13) for the fall-off behaviour of the physical field we gather from (5.13) that the data are trivial, i.e. determine a conformally flat solution of Einstein's equation's, only if $(\widetilde{M}, \tilde{g})$ is a space of negative constant curvature, i.e. only if $(M, h)$ is conformally flat. It follows that there exist non-trivial solutions to 
Einstein's equations satisfying (5.12). It may be remarked, however, that the conformal flatness of $(M, h)$ does not necessarily imply triviality of the solution. Consider the case where $M=\left\{x \in \mathbb{R}^{3}\left|r_{1} \leq\right| x \mid \leq r_{2}\right\}$ with real numbers $0<r_{1}<r_{2}$ and where $h$ is the metric induced on $M$ by the flat standard metric on $\mathbb{R}^{3}$. It is easy to see that the regularity condition $(5.12)$ is satisfied. The resulting space $(\widetilde{M}, \tilde{g})$ cannot be of constant negative curvature since it is complete and simply connected but $\widetilde{M}$ is not diffeomorphic to $\mathbb{R}^{3}$. Furthermore, it follows that the hyperboloidal data and consequently the solution of Einstein's equations $(\mathscr{C}, \gamma)$ determined from $(M, h)$ are spherically symmetric. By Birkhoff's Theorem $(\mathscr{l}, \gamma)$ represent thus a portion of a Schwarzschild-Kruskal space-time with positive mass. We also see from this example that in the Schwarzschild-Kruskal space-time there exist smooth spacelike hypersurfaces which extend from one future null infinity to the other future null infinity and on which the second fundamental form is proportional to the interior metric.

Acknowledgements. H.F. should like to thank the I.H.E.S., Paris, and the Royal Institute of Technology, Stockholm, for hospitality. P. T.C. acknowledges a collaboration with J. Jezierski at an early stage of work on this paper; he also wishes to thank J. Jezierski, G. Lysik, R. Mazzeo and B. Ziemian for useful discussions.

\section{References}

1. Andersson, L.: Elliptic systems on manifolds with asymptotically negative curvature. Submitted at Indiana Univ. Math. J. 1990

2. Andersson, L., Chruściel, P. T.: In preparation

3. Aviles, P., McOwen, R.C.: Complete conformal metrics with negative scalar curvature in compact Riemannian manifolds. Duke Math. J. 56, 395-398 (1988)

4. Aviles, P., McOwen, R.C.: Conformal deformation to constant negative scalar curvature on noncompact Riemannian manifolds. J. Differ. Geom. 27, 225-239 (1988)

5. Besse, A. L.: Einstein manifolds, Vol. 10. (Ergebnisse der Math. 3. Folge) Berlin, Heidelberg, New York: Springer 1987

6. Friedrich, H.: On static and radiative spacetimes. Commun. Math. Phys. 119, 51-73 (1988)

7. Friedrich, H.: On the global existence and the asymptotic behaviour of solutions to the EinsteinMaxwell-Yang-Mills equation. J. Differ. Geom. 34, 275-345 (1991)

8. Graham, C. R., Lee, J. M.: Einstein metrics with prescribed conformal infinity on the ball. Adv. Math. 87, 186-225 (1991)

9. Loewner, C., Nirenberg, L.: Partial differential equations invariant under conformal or projective transformations. In: Contributions to analysis. Ahlfors, L. V. et al. (eds.) New York: Academic Press 1974

10. Mazzeo, R.: Hodge cohomology of negatively curved manifolds. Technical report, 1986

11. Mazzeo, R.: The Hodge cohomology of conformally compact metrics. J. Differ. Geom. 28, 309-339 (1988)

12. Mazzeo, R.: Regularity for the singular Yamabe problem. Stanford University preprint, 1990

13. Mazzeo, R., Melrose, R. B.: Meromorphic extension of the resolvent on complete spaces with asymptotically negative curvature. J. Funct. Anal. 75, 260-310 (1987)

14. Melrose, R. B.: Transformation of boundary value problems. Acta Math. 147, 149-236 (1981)

15. Penrose, R.: Zero rest-mass fields including gravitation: asymptotic behaviour. Proc. R. Soc. Lond. A284, 159-203 (1965)

16. Schoen, R.M.:Conformal deformation of a Riemannian metric to constant scalar curvature.J. Differ. Geom. 20, 479-495 (1984) 